\title{
CONSTRUCTION OF CHARACTERISTIC FUNCTIONS FOR CLASSES OF INFINITELY DIFFERENTIABLE FUNCTIONS
}

\author{
M. AllOUCHE, R. COUTURE, AND C. T. TSAI
}

(Communicated by R. Daniel Mauldin)

\begin{abstract}
Characteristic functions are constructed for classes of infinitely differentiable functions defined on a half-line and for Paley-Wiener classes. A corresponding result is given for normal operators defined in Hilbert space.
\end{abstract}

\section{INTRODUCTION}

Let $M_{n}$ be a sequence of positive numbers. We denote by $C\left\{M_{n}\right\}$ the class of infinitely differentiable functions $f(x)$, real valued and satisfying the inequality

$$
\left|f^{(n)}(x)\right| \leq A B^{n} M_{n}, \quad n=0,1, \ldots,
$$

for all $x \in \mathbf{R}$ and some constants $A$ and $B$ (which may depend on $f$ ). According to Gorny's inequalities [6], we can replace the sequence $M_{n}$ by one that is logarithmically convex, and this, without altering the class $C\left\{M_{n}\right\}$. So now we assume the sequence $M_{n}$ to be logarithmically convex. Following Bang's terminology, we shall term a function $h(x)$ characteristic of the class $C\left\{M_{n}\right\}$ if it belongs to this class and if, for some positive constants $C$ and $D, C D^{n} M_{n} \leq \sup _{-\infty<x<\infty}\left|h^{(n)}(x)\right|, n \geq 0$. Gorny [6] was first to construct such functions. Using a method of Cartan [4], who dealt with this problem in the case of functions defined on a bounded interval, Bang [3] has given another construction of a characteristic function of $C\left\{M_{n}\right\}$. It can be written as

$$
h(x)=\sum_{k=0}^{\infty} \frac{1}{2^{k}} \frac{\cos \left(-\frac{1}{4}+r_{k} x\right)}{T\left(r_{k}\right)}, \quad-\infty<x<\infty,
$$

where $T(r)=\sup _{n \geq 0} r^{n} / M_{n}, r>0$, and $r_{k}$ is chosen so that $M_{k}=r_{k}^{k} / T\left(r_{k}\right)$ (Bang's choice of $r_{k}$ is $M_{k+1} / M_{k}$ ).

Our aim is to extend Cartan's method to other types of classes.

Received by the editors August 16, 1989.

1980 Mathematics Subject Classification (1985 Revision). Primary 26E10; Secondary 47B15.

Key words and phrases. Classes of infinitely differentiable functions, characteristic functions, normal operators. 


\section{The Classes $C_{d}\left\{M_{n}\right\}$}

The class $C_{d}\left\{M_{n}\right\}$ consists of those functions $f(x)$ defined and infinitely differentiable on the half-line $x \geq 0$, which satisfy (1.1). As a consequence of Gorny's inequalities, we have $C_{d}\left\{M_{n}\right\}=C_{d}\left\{M_{n}^{d}\right\}$. Here $M_{n}^{d}$ is the upper envelope of all sequences bounded by $M_{n}$ and of the form $a r^{n} / n^{n}, n \geq 0$, where $a$ and $r$ are nonnegative constants. We will assume that $M_{n}=M_{n}^{d}$. We construct first a characteristic function for $C_{d}\left\{M_{n}\right\}$ in the case $M_{n}=a r^{n} / n^{n}$ (in the case $C\left\{M_{n}\right\}$ where $M_{n}$ is logarithmically convex, this sequence $M_{n}$ is the upper envelope of sequences of the form $a r^{n}$; a characteristic function for $C\left\{a r^{n}\right\}$ is then $a \cos (-\pi / 4+r x)$; compare with (1.2); in all these subcases one must obtain the same constants $A, B, C$, and $D$ ).

Lemma 1. Let $a$ and $r$ be two positive constants. If $h_{a, r}(x)=a \cos (\sqrt{r x})$, $x \geq 0$, then

$$
\begin{gathered}
\left|h_{a, r}^{(n)}(x)\right| \leq e^{n}\left(a r^{n} / n^{n}\right), \quad n \geq 0, \quad x \geq 0, \\
h_{a, r}^{(n)}(0)=(-1)^{n} a r^{n} n ! /(2 n) !, \quad n \geq 0 .
\end{gathered}
$$

Proof. We can assume $a=r=1$. Then $h_{1,1}(x)=h(x)$, where $h(z)=$ $\sum_{n=0}^{\infty}(-z)^{n} /(2 n) !, z \in \mathbf{C}$, and (2.2) follows.

For $\rho>0$, let $\Omega_{\rho}=\{z=x+i y \in \mathbf{C}$ : either $|z|<\rho$, or $x>0$ and $|y|<\rho\}$. Now, the image of $\Omega_{\sqrt{\rho}}$ by the mapping $z \mapsto z^{2}$ contains $\Omega_{\rho}$ so, since $h\left(z^{2}\right)=\cos z$ and $|\cos z| \leq e^{|y|}$, we obtain

$$
\sup _{z \in \Omega_{\rho}}|h(z)| \leq e^{\sqrt{\rho}}, \quad \rho>0 .
$$

If we apply Cauchy's inequalities to $h(z)$ with a disk $D_{n^{2}}\left(x_{0}\right) \subset \Omega_{n^{2}}$ centered at $x_{0} \geq 0$ and with radius $n^{2}$ we obtain, using (2.3),

$$
\left|h^{(n)}\left(x_{0}\right)\right| \leq e^{n} n ! / n^{2 n} \leq e^{n} / n^{n}, \quad n \geq 0,
$$

and (2.1) follows.

Theorem 1. Let $M_{n}$ be a sequence of positive numbers with $M_{n}=M_{n}^{d}$. Then, there exists a function $h(x) \in C_{d}\left\{M_{n}\right\}$ such that, for some positive constants $C$ and $D$,

$$
C D^{n} M_{n} \leq\left|h^{(n)}(0)\right|, \quad n \geq 0 .
$$

Proof. If one defines $H(r)=\sup _{n \geq 0} r^{n} /\left(n^{n} M_{n}\right), r>0$ (see [1]) then, since $M_{n}=M_{n}^{d}$, we have

$$
M_{n}=\sup _{r>0} \frac{r^{n}}{H(r) n^{n}}, \quad n \geq 0 .
$$


For each $n$, let $r_{n}>0$ be a value of $r$ giving the supremum. We put (compare with (1.2))

$$
h(x)=\sum_{k=0}^{\infty} \frac{1}{2^{k}} \frac{\cos \left(\sqrt{r_{k} x}\right)}{H\left(r_{k}\right)}, \quad x \geq 0 .
$$

Using (2.1), we obtain for $n \geq 0$ and $x \geq 0$,

$$
\left|h^{(n)}(x)\right| \leq \sum_{k=0}^{\infty} \frac{1}{2^{k}} \frac{e^{n} r_{k}^{n}}{H\left(r_{k}\right) n^{n}} \leq 2 e^{n} M_{n},
$$

so that $h(x) \in C_{d}\left\{M_{n}\right\}$.

Also from (2.2) we have that the $n$th derivative at 0 of all terms in (2.5) are of the same sign, and so, for $n \geq 0$,

$$
\left|h^{(n)}(0)\right| \geq \frac{1}{2 n} \frac{r_{n}^{n} n !}{H\left(r_{n}\right)(2 n) !} \geq \frac{1}{4 n} \frac{r_{n}^{n}}{H\left(r_{n}\right) n^{n}}=\frac{1}{4^{n}} M_{n} .
$$

Remarks. The classes $C_{d}\left\{M_{n}\right\}$ are introduced by Mandelbrojt [9] in the context of a theory of generalized quasi-analyticity. Agmon [1] studied the problem of the equivalence (or, more generally, the inclusion) of such classes: $C_{d}\left\{M_{n}\right\} \subset$ $C_{d}\left\{L_{n}\right\}$ if and only if $M_{n}^{d} \leq A B^{n} L_{n}, n \geq 0$, for some constants $A$ and $B$. This follows from the equality $C_{d}\left\{M_{n}\right\}=C_{d}\left\{M_{n}^{d}\right\}$ and Theorem 1. However, Agmon's statement that $\underline{\lim } M_{n}^{1 / n}<\infty$ implies $C_{d}\left\{M_{n}\right\}=C_{d}\{1\}$ is not correct. For instance we have $C_{d}\left\{n^{-n}\right\} \neq C_{d}\{1\}$. Then he used Laguerre polynomials in conjunction with the condition $\underline{\lim } M_{n}^{1 / n}=\infty$. Mandelbrojt (see [10, p. 230]) also considered the question under the assumption $\underline{\lim } M_{n}^{1 / n}>0$.

The function $\cos (\sqrt{x})$ is a special case of the Mittag-Leffler functions $E_{\alpha}(z)$ (see, i.e. [11]). Korenbljum [7] had used these functions to show, in particular, that $C_{d}\left\{M_{n}\right\} \neq\{0\}$ if $\underline{\lim } n M_{n}^{1 / n}>0$.

\section{The Paley-Wiener classes}

For a sequence of positive numbers $M_{n}$ and for $1 \leq p<\infty$, let $L^{p}\left\{M_{n}\right\}$ denote the class of complex-valued, infinitely differentiable functions $f(x)$, $x \in \mathbf{R}$, which satisfy, for some constants $A$ and $B$,

$$
\left\{\int_{-\infty}^{\infty}\left|f^{(n)}(x)\right|^{p} d x\right\}^{1 / p} \leq A B^{n} M_{n}, \quad n \geq 0 .
$$

As in the $C\left\{M_{n}\right\}$ case, we can assume that the sequence $M_{n}$ is logarithmically convex (see, i.e., [13]).

Let $\varphi(\xi), \xi \in \mathbf{R}$, be a function of class $C^{2}$, nonnegative, not identically zero, and with compact support contained in $(-\infty, 0]$. We put

$$
\varphi_{r}(\xi)=\varphi(\xi-r), \quad h_{r}(x)=\hat{\varphi}_{r}(x)=\int_{-\infty}^{\infty} \varphi_{r}(\xi) e^{i x \xi} d \xi
$$


and

$$
h(x)=\sum_{k=0}^{\infty} \frac{1}{2^{k}} \frac{h_{r_{k}}(x)}{T\left(r_{k}\right)}, \quad x \in \mathbf{R},
$$

where $T(r)$ and $r_{k}$ are as in $\S 1$.

Lemma 2. Let $K>0$ be such that $\operatorname{supp} \varphi \subset[-K, 0]$. Then there exist constants $A$ and $B$ such that for all $r>0$ and $1 \leq p<\infty$,

$$
\left\|h_{r}^{(n)}\right\|_{p} \leq A(B \max (r, K))^{n}, \quad n \geq 0 .
$$

We also have, if $r>K$,

$$
\left\|h_{r}^{(n)}\right\|_{2} \geq \sqrt{2 \pi}\|\varphi\|_{2}(r-K)^{n}, \quad n \geq 0 .
$$

Proof. Since $h_{r}^{(n)}(x)$ is the Fourier transform of $(i \xi)^{n} \varphi_{r}(\xi)$, we have

$$
\left\|h_{r}^{(n)}\right\|_{\infty} \leq\left\|\xi^{n} \varphi_{r}(\xi)\right\|_{1} \leq r_{0}^{n}\|\varphi\|_{1}, \quad n \geq 0
$$

where $r_{0}=\max (r, K)$. Also $-x^{2} h_{r}^{(n)}(x)$ is the Fourier transform of

$$
d^{2}\left((i \xi)^{n} \varphi_{r}(\xi)\right) / d \xi^{2}
$$

and

$$
\begin{aligned}
\left\|x^{2} h_{r}^{(n)}(x)\right\|_{\infty} & \leq\left\|\frac{d^{2}}{d \xi^{2}}\left((i \xi)^{n} \varphi_{r}(\xi)\right)\right\|_{1} \\
& \leq n(n-1) r_{0}^{n-2}\|\varphi\|_{1}+2 n r_{0}^{n-1}\left\|\varphi^{\prime}\right\|_{1}+r_{0}^{n}\left\|\varphi^{\prime \prime}\right\|_{1} .
\end{aligned}
$$

It follows from (3.4) and (3.5) that for some constants $A_{1}$ and $B_{1},\left|h_{r}^{(n)}(x)\right|$ $\leq A_{1} B_{1}^{n} r_{0}^{n} \min \left(1, x^{-2}\right), x \in \mathbf{R}$. From this inequality, we obtain (3.2).

Now we assume $r>K$. By Plancherel's formula, we have

$$
\left\|h_{r}^{(n)}\right\|_{2}^{2}=2 \pi\left\|\xi^{n} \varphi_{r}(\xi)\right\|_{2}^{2} \geq 2 \pi(r-K)^{2 n}\|\varphi\|_{2}^{2}
$$

and obtain (3.3).

Lemma 3. Let $f(x), x \in \mathbf{R}$, be measurable. If $\|f\|_{1} \leq A$ and $\|f\|_{2} \geq B$ then $\|f\|_{p}>(A / B)^{2 / p}\left(B^{2} / A\right)$ for $p \geq 2$. If $\|f\|_{p} \leq A$ for all $p \geq 2$ and $\|f\|_{2} \geq B$, then $\|f\|_{p} \geq B$ for $1 \leq p \leq 2$.

Proof. By Hölder's inequality, $\log \|f\|_{p}^{p}$ is a convex function of $p$. The second statement of the lemma follows immediately. Also, if $\alpha=\log \left(B^{2} / A\right)$ and $\beta=\log (A / B)^{2}, \alpha p+\beta$ is the linear function of $p$, equal to $\log A$ at $p=1$ and to $\log B^{2}$ at $p=2$, and we obtain for $p \geq 2, \log \left(\left(B^{2} / A\right)^{p}(A / B)^{2}\right)=$ $\alpha p+\beta \leq \log \|f\|_{p}^{p}$. This gives the first statement.

Now we will show that $h(x)$, as given by (3.1), is characteristic of all the classes $L^{p}\left\{M_{n}\right\}, 1 \leq p<\infty$. 
Theorem 2. Let $M_{n}$ be a sequence of positive numbers, logarithmically convex. Then the function $h(x)$, as given by (3.1), belongs to $L^{p}\left\{M_{n}\right\}, 1 \leq p<\infty$. Also, for each such $p$, there exist positive constants $C$ and $D$ such that

$$
C D^{n} \leq\left\|h^{(n)}\right\|_{p}, \quad n \geq 0 .
$$

Proof. Let $K$ be as in Lemma 2. Then, using (3.2),

$$
\left\|h^{(n)}\right\|_{p} \leq A(B K)^{n} \sum_{r_{k}<K} \frac{1}{2^{k}} \frac{1}{T\left(r_{k}\right)}+A B^{n} \sum_{r_{k} \geq K} \frac{1}{2^{k}} \frac{r_{k}^{n}}{T\left(r_{k}\right)} .
$$

The first sum is bounded by a constant $D$ (dependent only on $K$ and the sequence $M_{n}$ ). Also, for some $\delta>0, \delta^{n} \leq M_{n}, n \geq 0$, since $M_{n}$ is logarithmically convex. We thus obtain, using the definition of $T(r)$ (see $\S 1$ ), $\left\|h^{(n)}\right\|_{p} \leq A D(B K / \delta)^{n} M_{n}+A B^{n} M_{n}$ and it follows that $h \in L^{p}\left\{M_{n}\right\}$.

By Lemma 3 it is sufficient to show (3.6) for $p=2$. First we assume that the sequence $M_{n}^{1 / n}$ is unbounded. Then $\underline{\lim } r_{k}=\infty$. Since it is also sufficient to prove (3.6) for large values of $n$, we may assume that $n$ is such that $r_{n}>2 K$. By Plancherel's formula and the positivity of $\varphi$, we obtain

$$
\begin{aligned}
\left\|h^{(n)}\right\|_{2}^{2} & \geq 2 \pi \int_{0}^{\infty}\left|\xi^{n} \sum_{k=0}^{\infty} \frac{1}{2^{k}} \frac{\varphi_{r_{k}}(\xi)}{T\left(r_{k}\right)}\right|^{2} d \xi \\
& \geq \frac{\pi}{2^{n-1}} \int_{0}^{\infty} \xi^{2 n}\left(\frac{\varphi_{r_{n}}(\xi)}{T\left(r_{n}\right)}\right)^{2} d \xi \\
& =\frac{\pi}{2^{n-1} T\left(r_{n}\right)^{2}}\left\|h_{r_{n}}^{(n)}\right\|_{2}^{2} .
\end{aligned}
$$

Now using (3.3), the inequality $r_{n}>2 K$ and the defining property of $r_{n}$ (see $\S 1$ ) we deduce that $\left\|h^{(n)}\right\|_{2}^{2} \geq \pi^{2} \|_{2}^{2} M_{n}^{2}$ and obtain (3.6).

Finally, we assume that the sequence $M_{n}^{1 / n}$ is bounded. It is then sufficient to verify (3.6) with $M_{n} \equiv 1$. By Plancherel's theorem and the positivity of $\varphi$, we have

$$
\left\|h^{(n)}\right\|_{2}^{2} \geq 2 \pi \int_{-\infty}^{\infty} \xi^{2 n} f(\xi) d \xi
$$

where $f(\xi) \geq 0$ is continuous and not identically zero. On some closed interval $I$ not containing zero, $f(\xi) \geq \delta>0$. So, if $D=\inf _{\xi \in I}|\xi|^{2}$, we obtain $\left\|f^{(n)}\right\|_{2}^{2} \geq 2 \pi \delta|I| D^{n}$ and (3.6) follows.

Remarks. The classes $L^{2}\left\{M_{n}\right\}$ were introduced by Paley and Wiener [12] in their study of the problem of quasi-analyticity by means of the Fourier transformation.

Mandelbrojt [8] proved the following statement. Let $M_{n}$ and $L_{n}$ be two sequences of positive numbers, and assume $M_{n}$ is logarithmically convex; then $L^{2}\left\{M_{n}\right\} \subset L^{2}\left\{L_{n}\right\}$ if and only if $M_{n} \leq A B^{n} L_{n}, n \geq 0$, provided $\log M_{n}=$ $O\left(n^{2}\right)$. The statement follows immediately, without this last condition, from 
Theorem 2. Using a topological method of Hormander, Couture [5] solved the problem of the equivalence of the classes $L^{p}\left\{M_{n}\right\}, 1 \leq p<\infty$ (same statement as for $p=2$ ). This also follows from Theorem 2 .

\section{THE CLASSES $H^{T}\left\{M_{n}\right\}$}

Let $H$ be a complex Hilbert space and $T$, densely defined (possibly unbounded) normal operator in $H$. Let $M_{n}$ be a sequence of positive numbers. We define $H^{T}\left\{M_{n}\right\}$ as the "class" of vectors $v \in H$ belonging to the domain of definition of all iterates $T^{n}$ of $T$ and satisfying

$$
\left\|T^{n} v\right\| \leq A B^{n} M_{n}, \quad n \geq 0,
$$

for some constants $A$ and $B$. Since $\left\|T^{n} v\right\|$ is a logarithmically convex sequence (see, for instance the appendix in [2]), we may assume the same of $M_{n}$. If we take, for example, $H=L^{2}(\mathbf{R})$ and $T=d / d x$ we have $H^{T}\left\{M_{n}\right\}=$ $L^{2}\left\{M_{n}\right\}$.

Let $d P$ denote the spectral decomposition of $T$, and $P_{r, s}, r<s$, the subspace of $H$ corresponding by $d P$ to the ring $\{z \in \mathbf{C}: r<|z| \leq s\}$.

Lemma 4. If $v \in P_{r, s}$ has norm $\|v\|=1$, then $r^{n} \leq\left\|T^{n} v\right\| \leq s^{n}, n \geq 0$.

Proof. We have for all $n \geq 0$,

$$
\left\|T^{n} v\right\|=\int_{\mathbf{C}}|\lambda|^{2 n}\left\|d P_{\lambda} v\right\|^{2}=\int_{r<|\lambda| \leq s}|\lambda|^{2 n}\left\|d P_{\lambda} v\right\|^{2} .
$$

The lemma follows.

Theorem 3. We assume that there exists a constant $\delta, 0<\delta<1$, such that $P_{\delta r, r} \neq\{0\}$ if $r$ is sufficiently large. Then there exists a characteristic vector $v$ for the class $H^{T}\left\{M_{n}\right\}$; that is, $v \in H^{T}\left\{M_{n}\right\}$ and, for some positive constants $C$ and $D, C D^{n} \leq\left\|T^{n} v\right\|, n \geq 0$.

Proof. Take $r_{0}$ so that $r \geq r_{0}$ implies $P_{r}=P_{\delta r, r} \neq\{0\}$. First let us assume that the sequence $M_{n+1} / M_{n}$ is unbounded. We choose two sequences of real numbers, $k_{i}$ and $r_{i}, i \geq 1$, such that

(i) $r_{i}>r_{0}$ and $k_{i}$ are positive integers;

(ii) $M_{k_{i}}=r_{i}^{k_{i}} / T\left(r_{i}\right)($ see $\S 1)$;

(iii) $k_{i+1}$ is the smallest integer $k$ such that $\delta^{-1} r_{i} \leq M_{k} / M_{k-1}, i \geq 1$.

Condition (ii) implies $M_{k_{i}} / M_{k_{i}-1} \leq r_{i}$ so, $k_{i+1}>k_{i}$ and $r_{i+1} \geq \delta^{-1} r_{i}$ by (iii). The sequence $k_{i}$ is thus strictly increasing and the rings $\left\{\lambda \in \mathbf{C}: \delta r_{i}<\right.$ $\left.|\lambda|<r_{i}\right\}, i \geq 1$, are disjoint. The corresponding subspaces $P_{r_{i}}$ are then mutually orthogonal. By (i), $P_{r_{i}} \neq\{0\}$ and we choose a vector $v_{i} \in P_{r_{i}}$ with 
$\left\|v_{i}\right\|=1$. We then put

$$
v=\sum_{i=1}^{\infty} \frac{1}{2^{k_{i}}} \frac{v_{i}}{T\left(r_{i}\right)} .
$$

As $T^{n} v_{i} \in P_{r_{i}}$, the vectors $T^{n} v_{i}, i \geq 1$, are mutually orthogonal and we have

$$
\left\|T^{n} v\right\|^{2}=\sum_{i=1}^{\infty} \frac{1}{2^{2 k_{i}}} \frac{\left\|T^{n} v_{i}\right\|^{2}}{T\left(r_{i}\right)^{2}}, \quad n \geq 0 .
$$

By Lemma 4, $\left\|T^{n} v\right\|^{2} \leq \sum_{i=1}^{\infty}\left(1 / 2^{2 k_{i}}\right)\left(r_{i}^{2 n} / T\left(r_{i}\right)^{2}\right)$ and so, using the definition of $T(r)$ (see $\S 1),\left\|T^{n} v\right\| \leq\left\{\sum_{i=1}^{\infty} 1 / 2^{2 k_{i}}\right\}^{1 / 2} M_{n}, n \geq 0$. We have shown that $v \in H^{T}\left\{M_{n}\right\}$.

On the other hand, given an integer $n \geq k_{1}$, we take $i$ as the greatest integer such that $k_{i} \leq n$ and using (4.1) we obtain Lemma 4 and (ii),

$$
\left\|T^{n} v\right\| \geq \frac{1}{2^{2 k_{i}}} \frac{\left(\delta r_{i}\right)^{n}}{T\left(r_{i}\right)} \geq \frac{r_{i}^{n-k_{i}}}{\left(4 \delta^{-1}\right)^{n}} M_{k_{i}} .
$$

Since $n<k_{i+1}$, by (iii) we have $M_{n} / M_{n-1} \leq \delta^{-1} r_{i}$ and so, using logarithmic convexity, $M_{n} \leq M_{k_{i}}\left(\delta^{-1} r_{i}\right)^{n-k_{i}}$. Combining this inequality with (4.2) we obtain $\left\|T^{n} v\right\| \geq\left(\delta^{2} / 4\right)^{n} M_{n}$, and $v$ satisfies the requirements of the theorem.

Finally, if the sequence $M_{n+1} / M_{n}$ is bounded, one may simply choose $r$ so that $P_{r} \neq\{0\}$ and take $v$ equal to a vector of norm 1 in $P_{r}$. Using Lemma 4, one easily obtains that this vector $v$ is characteristic of the class $H^{T}\left\{M_{n}\right\}$.

\section{REFERENCES}

1. S. Agmon, Sur l'équivalence des classes de fonctions indéfiniment dérivables sur un demi-axe, C.R. Acad. Sci. Paris 230 (1950), 350-352.

2. M. Allouche, Fonctions caractéristiques de certaines classes de fonctions indéfiniment dérivables, Thesis, Québec, 1986.

3. T. Bang, Om quasi-analytiske funktioner, Thesis, Copenhagen, 1946.

4. H. Cartan, Sur les maxima des dérivées successives d'une fonction, C.R. Acad. Sci. Paris 210 (1940), 431-434.

5. R. Couture, Classes de fonctions $C^{\infty}$ et approximation par des sommes d'exponentielles, Thesis, Québec, 1980.

6. A. Gorny, Contribution à l'étude des fonctions dérivables d'une variable réelle, Acta Math. 71 (1939), 317-358.

7. V. I. Korenbljum, Conditions of nontriviality of certain classes of functions analytic in a sector, and problems of quasianalyticity, Soviet Math. Dokl. 7 (1966), 232-236.

8. S. Mandelbrojt, Sur l'équivalence de deux classes de fonctions de M. M. Paley et Wiener, C.R. Acad. Sci. Paris 204 (1937), 1860-1863.

9. __ Sur une inégalité fondamentale, Ann. Ecole Norm. Sup. (3) 63 (1946), 351-378.

10. Séries adhérentes, régularisation des suites, applications, Gauthier Villars, Paris, 1952.

11. G. Mittag-Leffler, Sur la représentation arithmétique des fonctions analytiques d'une variable complexe, Internat. Congress, 1908, pp. 67-85. 
12. R.E.A.C. Paley and N. Wiener, Notes on the theory and application of Fourier transforms. I-II, Trans. Amer. Math. Soc. 35 (1933), 348-355.

13. E. M. Stein, Functions of exponential type, Ann. of Math. (2) 65 (1957), 582-592.

Saint-Jean Faculty, University of Alberta, Edmonton, Alberta, T6C 4G9, Canada

Department of Mathematics, Laval University, Québec, G1K 7P4, Canada

Department of Mathematics, Bishop's University, Lennoxville, Québec, J1M $1 Z 7$, CANADA 